\title{
Multiple Petersen subdivisions in permutation graphs*
}

\author{
Tomáš Kaiser ${ }^{\dagger}$ \\ Department of Mathematics \\ and Institute for Theoretical Computer Science \\ University of West Bohemia \\ Univerzitní 8, 30614 Plzeň, Czech Republic \\ kaisert@kma.zcu.cz \\ Jean-Sébastien Sereni ${ }^{\ddagger}$ \\ Zelealem B. Yilma ${ }^{\ddagger}$ \\ CNRS \\ LIAFA, Univ. Paris Diderot \\ 175 rue du Chevaleret, 75013 Paris, France \\ sereni@kam.mff.cuni.cz \\ zelealemby@gmail.com
}

Submitted: Apr 9, 2012; Accepted: Feb 18, 2013; Published: Feb 25, 2013

Mathematics Subject Classifications: 05C99, 05C75

\begin{abstract}
A permutation graph is a cubic graph admitting a 1-factor $M$ whose complement consists of two chordless cycles. Extending results of Ellingham and of Goldwasser and Zhang, we prove that if $e$ is an edge of $M$ such that every 4-cycle containing an edge of $M$ contains $e$, then $e$ is contained in a subdivision of the Petersen graph of a special type. In particular, if the graph is cyclically 5-edge-connected, then every edge of $M$ is contained in such a subdivision. Our proof is based on a characterization of cographs in terms of twin vertices. We infer a linear lower bound on the number of Petersen subdivisions in a permutation graph with no 4-cycles, and give a construction showing that this lower bound is tight up to a constant factor.
\end{abstract}

Keywords: graph theory; permutation graph; Petersen subdivision; cograph

*Supported by project MEB 021115 of the Czech Ministry of Education and PHC Barrande 24444XD of the French MAE. This work falls within the scope of the LEA STRUCO.

${ }^{\dagger}$ Partially supported by project P202/12/G061 of the Czech Science Foundation.

¥This author’s work was partially supported by the French Agence Nationale de la Recherche under reference ANR 10 JCJC 020401. 


\section{Introduction}

A special case of Tutte's 4-flow conjecture [7] states that every bridgeless cubic graph with no minor isomorphic to the Petersen graph is 3-edge-colourable. Before this special case was shown to be true by Robertson et al. (cf. [6]), one of the classes of cubic graphs for which the conjecture was known to hold was the class of permutation graphs - i.e., graphs with a 2-factor consisting of two chordless cycles. Indeed, by a result of Ellingham [3], every permutation graph is either Hamiltonian - and hence 3-edgecolourable - or contains a subdivision of the Petersen graph. To state his theorem more precisely, we introduce some terminology.

Rephrasing the above definition, a cubic graph $G$ is a permutation graph if it contains a perfect matching $M$ such that $G-E(M)$ is the disjoint union of two cycles, none of which has a chord in $G$. A perfect matching $M$ with this property is called a distinguished matching in $G$. For brevity, if $G$ is a permutation graph with a distinguished matching $M$, then the pair $(G, M)$ is referred to as a marked permutation graph.

We let $P_{10}$ be the Petersen graph. Given a distinguished matching $M$ in $G$, an $M$-copy of $P_{10}$ is a subgraph $G^{\prime}$ of $G$ isomorphic to a subdivision of $P_{10}$ and composed of the two cycles of $G-E(M)$ together with five edges of $M$. Following Goldwasser and Zhang [4], an $M$-copy of $P_{10}$ is also referred to as an $M$ - $P_{10}$. Furthermore, an $M$-copy of the 4-cycle $C_{4}$ (or an $M-C_{4}$ ) is a 4-cycle in $G$ using two edges of $M$.

The proof of Ellingham's result implies that if a marked permutation graph $(G, M)$ contains no $M-P_{10}$, then it contains an $M-C_{4}$ (and is therefore Hamiltonian). Goldwasser and Zhang [4] obtained a slight strengthening:

Theorem 1. If $(G, M)$ is a marked permutation graph, then $G$ contains either two $M$ copies of $C_{4}$, or an $M$-copy of $P_{10}$.

Lai and Zhang [5] studied permutation graphs satisfying a certain minimality condition and proved that in a sense, they contain 'many' subdivisions of the Petersen graph.

The main result of this note is the following generalization of Theorem 1 .

Theorem 2. Let $(G, M)$ be a marked permutation graph on at least six vertices and let $e \in E(M)$. If $e$ is contained in every $M-C_{4}$ of $G$, then $e$ is contained in an $M$-copy of $P_{10}$.

Theorem 2 is established in Section 2. The proof is based on a relation between $M$-copies of $P_{10}$ in permutation graphs and induced paths in a related class of graphs.

Of particular interest is the corollary for cyclically 5-edge-connected graphs, that is, graphs containing no edge-cut of size at most 4 whose removal leaves at least two non-tree components.

Corollary 3. Every edge of a cyclically 5-edge-connected marked permutation graph $(G, M)$ is contained in an $M-P_{10}$. 


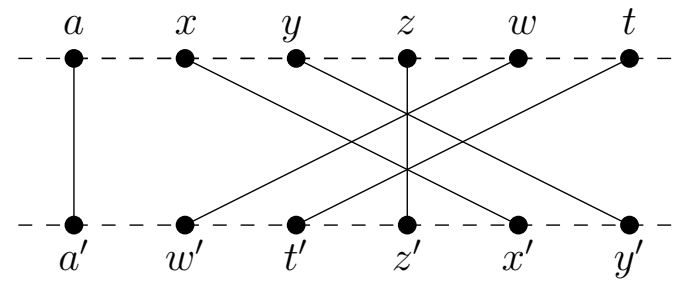

(a)

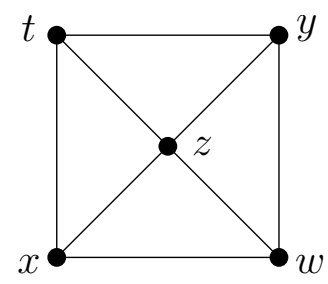

(b)

Figure 1: (a) The standard drawing of the graph $G$. (b) The corresponding graph $H_{a}$.

The class of cyclically 5-edge-connected permutation graphs is richer than one might expect. Indeed, it had been conjectured [8] that every cyclically 5-edge-connected permutation graph is 3-edge-colourable, but this conjecture has been recently disproved [2, Observation 4.2].

Theorem 2 readily implies a lower bound on the number of $M$-copies of the Petersen graph in a marked permutation graph $(G, M)$ such that $G$ contains no $M-C_{4}$ and has $n$ vertices. We improve this lower bound in Section 3. We also show that the bounds (which are linear in $n$ ) are optimal up to a constant factor.

We close this section with some terminology. If $G$ is a graph and $X \subseteq V(G)$, then $G[X]$ is the induced subgraph of $G$ on $X$. The set of all neighbours of a vertex $v$ of $G$ is denoted by $N_{G}(v)$.

\section{Proof of Theorem 2}

Let $(G, M)$ be a marked permutation graph. If $v \in V(G)$, then we write $v^{\prime}$ for the neighbour of $v$ in $M$ (which we call the friend of $v$ ). We extend this notation to arbitrary sets of vertices of $G$ : if $X \subseteq V(G)$, then we set

$$
X^{\prime}=\left\{v^{\prime}: v \in X\right\} .
$$

Let $A$ be the vertex set of one component of $G-E(M)$. Thus, $A^{\prime}$ is the vertex set of the other component and both $G[A]$ and $G\left[A^{\prime}\right]$ are chordless cycles.

In this section, we prove Theorem 2. Fix an edge $e$ of the matching $M$. Let $a$ and $a^{\prime}$ be its end-vertices. We also choose an orientation for each of the cycles $G[A]$ and $G\left[A^{\prime}\right]$. All these will be fixed throughout this section.

If $X \subseteq A$, then $G\{X\}$ is the spanning subgraph of $G$ obtained by adding to $G-E(M)$ all the edges $v v^{\prime}$, where $v \in X$. In expressions such as $G\{\{a, b\}\}$, we omit one pair of set brackets, and write just $G\{a, b\}$.

The auxiliary graph $H_{a}$ (with respect to the vertex $a$ ) is defined as follows. The vertex set of $H_{a}$ is $A-\{a\}$. Two vertices $x$ and $y$ of $H_{a}$ are adjacent in $H_{a}$ whenever the cyclic order of $a, x$ and $y$ on $G[A]$ is axy and the cyclic order of their friends on $G\left[A^{\prime}\right]$ is $a^{\prime} y^{\prime} x^{\prime}$.

Alternatively, consider the following standard procedure, illustrated in Figure 1. Arrange the vertices of $A$ on a horizontal line in the plane, starting on the left with $a$ and 


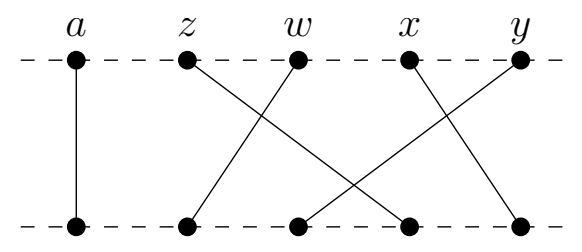

Figure 2: The graph $G\{a, x, y, z, w\}$ in Case 1 of the proof of Lemma 4 .

continuing along the cycle $G[A]$ according to the fixed orientation. Place the vertices of $A^{\prime}$ on another horizontal line, putting $a^{\prime}$ leftmost and continuing in accordance with the orientation of $G\left[A^{\prime}\right]$. Join each vertex $z \in A$ with its friend by a straight line segment. The segment $a a^{\prime}$ is not crossed by any other segment, and for $x, y \in A-\{a\}$, the segments $x x^{\prime}$ and $y y^{\prime}$ cross each other if and only if $x$ and $y$ are adjacent in $H_{a}$. Thus, $H_{a}$ can be directly read off the resulting figure, which is called the standard drawing of $G$.

A similar construction, without fixing the vertex $a$, gives rise to a class of graphs also called 'permutation graphs' (see [1]). In this paper, we only use this term as defined in Section 1.

The following lemma provides a link between induced paths in $H_{a}$ and $M$-copies of $P_{10}$ in $G$.

Lemma 4. Suppose that $H_{a}$ contains an induced path xyzw on 4 vertices. Then the subgraph $G\{a, x, y, z, w\}$ is an $M-P_{10}$ in $G$.

Proof. Let $P$ be the path $x y z w$ in $H_{a}$. Since $x y \in E(P)$, the edges $x x^{\prime}$ and $y y^{\prime}$ cross. By symmetry, we may assume that $x \in a C y$ and $y^{\prime} \in a^{\prime} C^{\prime} x^{\prime}$. First, note that $z \notin y C a$. Otherwise, as $z z^{\prime}$ crosses $y y^{\prime}$, it would follow that $z z^{\prime}$ also crosses $x x^{\prime}$, which contradicts the assumption that $x$ and $z$ are not adjacent in $G$. We now consider two cases, regarding whether or not $z \in a C x$.

Case 1: $z \in a C x$. Since the edges $z z^{\prime}$ and $x x^{\prime}$ do not cross, $z^{\prime} \in a^{\prime} C^{\prime} x^{\prime}$; moreover, since $z z^{\prime}$ and $y y^{\prime}$ cross, it follows that $z^{\prime} \in y^{\prime} C^{\prime} x^{\prime}$.

We assert that $w \in z C x$. Suppose that this is not the case. If $w \in a C z$, then $w w^{\prime}$ cannot cross $z z^{\prime}$ without crossing $y y^{\prime}$, contradicting the fact that $z w \in E(P)$ and $y w \notin E(P)$. If $w \in x C y$, then $w w^{\prime}$ crosses $x x^{\prime}$ or $y y^{\prime}$ regardless of the position of $w^{\prime}$, which results in a similar contradiction. Finally, if $w \in y C a$, then $w w^{\prime}$ cannot cross $z z^{\prime}$ without crossing $x x^{\prime}$.

Thus, we have shown that $w \in z C x$, which implies that $w^{\prime} \in a^{\prime} C^{\prime} y^{\prime}$, as $w w^{\prime}$ and $y y^{\prime}$ do not cross. Summing up, $G\{a, x, y, z, w\}$ is precisely as in Figure 2 and constitutes an $M$-copy of the Petersen graph.

Case 2: $z \notin a C x$. Then, $z \in x C y$. Since $z z^{\prime}$ and $x x^{\prime}$ do not cross, $z^{\prime} \in x^{\prime} C^{\prime} a^{\prime}$. As $w w^{\prime}$ crosses $z z^{\prime}$ but none of $x x^{\prime}$ and $y y^{\prime}$, the only possibility is that $w \in y C a$ and $w^{\prime} \in x^{\prime} C^{\prime} z^{\prime}$, which again produces an $M-P_{10}$.

By Lemma 4 , if there is no $M$-copy of $P_{10}$ containing $a a^{\prime}$ in $G$, then $H_{a}$ contains no induced path on 4 vertices. Such graphs are known as cographs or $P_{4}$-free graphs. There are various equivalent ways to describe them, summarized in the survey [1, Theorem 11.3.3] 


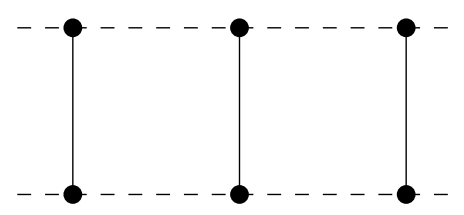

Figure 3: The triangular prism $G$ with the unique 1-factor $M$ such that $(G, M)$ is a marked permutation graph.

by Brandstädt, Le and Spinrad. We use the characterisation that involves pairs of twin vertices. (Two vertices $x$ and $y$ of a graph $H$ are twins if $N_{H}(x)=N_{H}(y)$.)

Theorem 5. A graph $G$ is $P_{4}$-free if and only if every induced subgraph of $G$ with at least two vertices contains a pair of twins.

To be able to use Lemma 4 in conjunction with Theorem 5, we need to interpret twin pairs of $H_{a}$ in terms of $G$.

Lemma 6. Let $x$ and $y$ be twins in $H_{a}$. Let $Q^{\prime}$ be the path in $G$ defined by

$$
Q^{\prime}= \begin{cases}x^{\prime} C^{\prime} y^{\prime} & \text { if } x y \notin E\left(H_{a}\right), \\ y^{\prime} C^{\prime} x^{\prime} & \text { otherwise. }\end{cases}
$$

Then $M$ matches the vertices of the path $x C y$ to those of $Q^{\prime}$ and vice versa.

Proof. Suppose, on the contrary, that the statement does not hold. By symmetry, we may assume that $x \in a C y$, and that $M$ contains an edge $w w^{\prime}$ with $w \in V(x C y)$ and $w^{\prime} \notin V\left(Q^{\prime}\right)$. We assert that $w w^{\prime}$ crosses exactly one edge from $\left\{x x^{\prime}, y y^{\prime}\right\}$. To prove this, we consider two cases according to whether or not $x x^{\prime}$ and $y y^{\prime}$ cross. If they do not cross, then $w w^{\prime}$ crosses only $x x^{\prime}$ (if $w^{\prime} \in V\left(a^{\prime} C^{\prime} x^{\prime}\right)$ ) or only $y y^{\prime}$ (if $w^{\prime} \in V\left(y^{\prime} C^{\prime} a^{\prime}\right)$ ). Otherwise, $w w^{\prime}$ crosses only $x x^{\prime}$ (if $w^{\prime} \in V\left(a^{\prime} C^{\prime} y^{\prime}\right)$ ) or only $y y^{\prime}$ (if $w^{\prime} \in V\left(x^{\prime} C^{\prime} a^{\prime}\right)$ ). In each case, we obtain a contradiction with the assumption that $x$ and $y$ are twins in $H_{a}$.

We now prove Theorem 2, proceeding by induction on the number of vertices of $G$. The base case is the triangular prism, the unique permutation graph on 6 vertices (Figure 3), for which the theorem is trivially true since $e$ cannot be contained in every $M-C_{4}$ of $G$. Therefore, we assume that $G$ has at least 8 vertices and that every $M$-copy of $C_{4}$ in $G$ contains the edge $a a^{\prime}$.

Suppose first that $a z z^{\prime} a^{\prime}$ is such an $M$-copy of $C_{4}$. Let $G_{0}$ be the cubic graph obtained by removing the edge $z z^{\prime}$ and suppressing the resulting degree 2 vertices $z$ and $z^{\prime}$. Set $M_{0}=M \backslash\left\{z z^{\prime}\right\}$. All $M_{0}$-copies of $C_{4}$ created by this operation contain the edge $a a^{\prime}$. Therefore, regardless of whether or not $G_{0}$ contains an $M_{0^{-}} C_{4}$, the induction hypothesis implies that $a a^{\prime}$ is contained in an $M_{0}$-copy of $P_{10}$. This yields an $M$-copy of $P_{10}$ in $G$ containing $a a^{\prime}$, as required.

Consequently, it may be assumed that $G$ does not contain any $M-C_{4}$. Assume that $H_{a}$ contains no pair of twin vertices. Theorem 5 implies that $H_{a}$ is not $P_{4}$-free. Let $X$ 
be a subset of $V\left(H_{a}\right)$ of size 4 such that $H_{a}[X] \simeq P_{4}$. By Lemma $4, G\{X \cup\{a\}\}$ is an $M$-copy of $P_{10}$, and the sought conclusion follows.

Thus, we may assume that $H_{a}$ contains twin vertices $x$ and $y$. Without loss of generality, $x$ belongs to $a C y$.

Let the path $Q^{\prime}$ be defined as in Lemma 6 . Thus, since $x$ and $y$ are twins in $H_{a}$, vertices of the path $x C y$ are only adjacent in $M$ to vertices of $Q^{\prime}$ and vice versa. We transform $G$ into another cubic graph $G_{1}$ by removing all vertices that are not contained in $\left\{a, a^{\prime}\right\} \cup V\left(x C y \cup Q^{\prime}\right)$ and adding the edges $a x, a y, a^{\prime} x^{\prime}$ and $a^{\prime} y^{\prime}$ (if they are not present yet). Let $M_{1}$ be the perfect matching of $G_{1}$ consisting of all the edges of $M$ contained in $G_{1}$. Note that although the transformation may create $M$-copies of $C_{4}$ not present in $G$, the edge $a a^{\prime}$ is contained in every $M_{1}$-copy of $C_{4}$ in $G_{1}$. Furthermore, the path $y C x$ in $G$ must have some internal vertices other than $a$, since otherwise $G$ would contain an $M-C_{4}$, namely $y a a^{\prime} y^{\prime}$. Thus, $G_{1}$ has fewer vertices than $G$. The induction hypothesis implies that $a a^{\prime}$ is contained in an $M_{1}$-copy of $P_{10}$ in $G_{1}$, and therefore also in $G$.

\section{Counting the Petersen copies}

Turning to the quantitative side of the question studied in Section 2, we now derive from Theorem 2 a lower bound on the number of $M$-copies of $P_{10}$ in a permutation graph with no $M-C_{4}$. The bound is linear in the order of the graph. We give a construction showing that this lower bound is tight up to a constant factor.

Throughout this section, $(G, M)$ is a marked permutation graph with vertex set $A \cup A^{\prime}$ just like in Section 2.

We will need two lemmas, the second of which we find to be of interest in its own right. The first lemma is an observation on auxiliary graphs which follows readily from the definition; its proof is omitted.

Lemma 7. Let $a, b \in A$. Then the following hold for each $x, y \in A-\{a, b\}$ :

(i) $a x \in H_{b}$ if and only if $b x \in H_{a}$,

(ii) $x y \in H_{b}$ if and only if $\left|\{b x, b y, x y\} \cap H_{a}\right| \in\{1,3\}$.

Lemma 8. Let $a, b \in A$. One of the following conditions holds:

- there is some $M-P_{10}$ in $G$ containing both $a a^{\prime}$ and $b b^{\prime}$, or

- for any $F \subset A$ with $|F|=4$ and $\{a, b\} \cap F=\emptyset$, it holds that $G\{F \cup\{a\}\} \simeq P_{10}$ if and only if $G\{F \cup\{b\}\} \simeq P_{10}$.

Proof. Assume that there exists no $M-P_{10}$ containing both $a a^{\prime}$ and $b b^{\prime}$. By Lemma 4 , it is sufficient to show that a set $\{u, w, x, y\} \subseteq A \backslash\{a, b\}$ induces a path of length 4 in $H_{a}$ if and only if it induces a path of length 4 in $H_{b}$.

Let $U_{1}=N_{H_{a}}(b)=N_{H_{b}}(a)$ and $U_{2}=A \backslash\left(U_{1} \cup\{a, b\}\right)$. Lemma 4 implies that in the auxiliary graph $H_{a}$, there is no induced path of length 4 containing the vertex $b$. Therefore, 
(i) if $x, y \in U_{1}, z \in U_{2}$, and $x y \notin H_{a}$, then $x z \in H_{a}$ if and only if $y z \in H_{a}$, and

(ii) if $x, y \in U_{2}, z \in U_{1}$, and $x y \in H_{a}$, then $x z \in H_{a}$ if and only if $y z \in H_{a}$.

Hence, if uwxy is an induced path of length 4 in $H_{a}$, then $\{u, w, x, y\} \cap U_{1}$ belongs to $\{\{u, w, x, y\},\{w, x\}, \emptyset\}$. By Lemma 7 , it follows that $\{u, w, x, y\}$ induces a path of length 4 in $H_{b}$ as well. More precisely, this path is uwxy if $\{u, w, x, y\} \cap U_{1} \in\{\{u, w, x, y\}, \emptyset\}$, and $u x w y$ if $\{u, w, x, y\} \cap U_{1}=\{w, x\}$. The conclusion follows by symmetry of the roles played by $a$ and $b$.

We can now prove the aforementioned lower bound.

Proposition 9. If $(G, M)$ is a marked permutation graph with $n \geqslant 40$ vertices and no $M-C_{4}$, then $(G, M)$ contains at least $n / 2-4 M$-copies of the Petersen graph.

Proof. If each edge of $M$ is contained in at least $5 M$-copies of $P_{10}$, the total number of copies is at least $(5 n / 2) / 5=n / 2$. Hence, we may assume that there exists $x \in\{1,2,3,4\}$ and an edge $e \in E(M)$ that is contained in only $x M$-copies of $P_{10}$. Let $\mathcal{C}$ be the set of these copies.

At least $n / 2-4 x-1$ edges of $M$ are not contained in any $M-P_{10}$ containing $e$. By Lemma 8 , if we replace $e$ by any such edge in any $M-P_{10}$ from $\mathrm{C}$, we obtain an $M-P_{10}$ again. These replacements yield $x(n / 2-4 x-1)$ distinct $M$-copies of $P_{10}$. Thus, in total, $(G, M)$ contains at least $x(n / 2-4 x)$ distinct $M$-copies of $P_{10}$. Minimizing this expression over $x \in\{1,2,3,4\}$ and using the assumption that $n \geqslant 40$, we deduce that the number of copies is at least $n / 2-4$, as asserted.

We now construct a family of marked permutation graphs $\left(G_{k}, M_{k}\right)$ showing that the linear estimate in Proposition 9 is tight up to a constant factor. The graph $G_{k}$ has $6 k+14$ vertices, contains no $M_{k}-C_{4}$, and the number of $M_{k}$-copies of the Petersen graph in $G_{k}$ is only $6 k+6$. (We note that graphs with a somewhat similar structure are constructed in $[4$, Section 3].)

For $k=4$, the graph $\left(G_{k}, M_{k}\right)$ is shown in Figure 4. We now give a formal definition and determine the number of $M_{k}$-copies of $P_{10}$.

Let $A=\{1,2, \ldots, 3 k+7\}$ and $A^{\prime}=\{\overline{1}, \overline{2}, \ldots, \overline{3 k+7}\}$. The vertex set of $G_{k}$ is $A \cup A^{\prime}$. On each of $A$ and $A^{\prime}$, we consider the standard linear order (in particular, $\overline{1}<\overline{2}<\cdots<\overline{3 k+7}$ ). As in Section 2, we write $i^{\prime}$ for the neighbour in $M_{k}$ of a vertex $i \in A$. Thus, $i^{\prime}=\bar{j}$ for a suitable $j$.

Let

$$
\begin{aligned}
E_{1}= & (2 i-1) \bar{i}: 1 \leqslant i \leqslant k\} \cup\{(2 k+i+3) \overline{(k+2 i+3)}: 1 \leqslant i \leqslant k\}, \\
E_{2}= & \{(2 i) \overline{(3 k+4-2 i)}: 1 \leqslant i \leqslant k-1\}, \\
E_{3}=\{(2 k) \overline{(k+2)},(2 k+1) \overline{(k+4)},(2 k+2) \overline{(k+1)},(2 k+3) \overline{(k+3)}, \overline{(3 k) \overline{(3 k+4)}},(3 k+7) \overline{(3 k+6)}\} . & (3 k+4) \overline{(3 k+5)},(3 k+5) \overline{(3 k+7)},(3 k+6) \overline{(3 k},
\end{aligned}
$$

Edges in $E_{1}, E_{2}$ and $E_{3}$ will be called vertical, skew and special, respectively. Moreover, the first four and the last four edges in $E_{3}$ are two groups of special edges. 


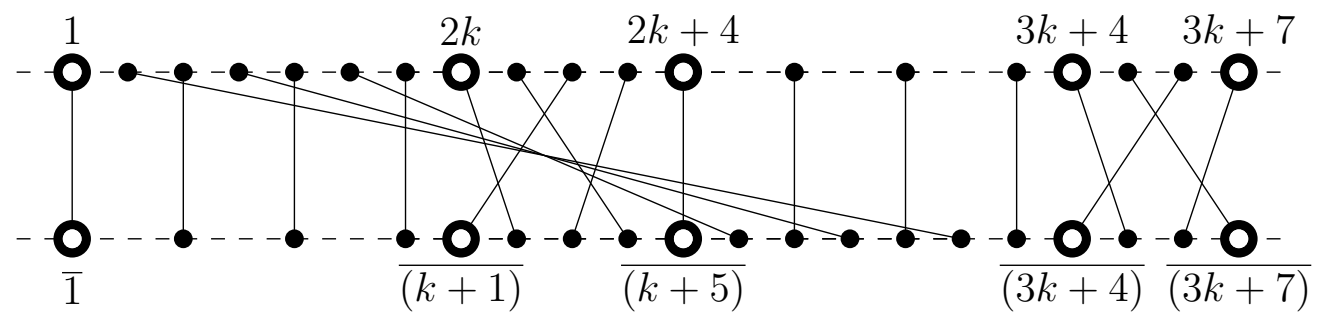

Figure 4: The marked permutation graph $\left(G_{4}, M_{4}\right)$. Labels are given only for the circled vertices.

Proposition 10. The marked permutation graph $\left(G_{k}, M_{k}\right)$ contains exactly $6 k+6 M$ copies of the Petersen graph.

Proof. Each of the groups of special edges forms an $M_{k}-P_{10}$ with each of the remaining $3 k+3$ edges of $M_{k}$. We prove that besides these $6 k+6$ copies, there are no other $M_{k}$-copies of $P_{10}$ in $G_{k}$.

For $X \subseteq M_{k}$, we let $G_{X}$ be the graph obtained from $G_{k}-M_{k}$ by adding the edges in $X$ and suppressing the degree 2 vertices.

Let $X$ be a subset of $M_{k}$ that contains no group of special edges. Suppose that $G_{k}\{X\}$ is isomorphic to the Petersen graph. To obtain a contradiction, we show that $G_{k}\{X\}$ contains a 4-cycle.

First of all, if $X$ contains a special edge, then it contains no other special edge from the same group. Indeed, a quick case analysis shows that if $Y$ consists of any two or three special edges in the same group, then $G_{Y}$ contains a $Y-C_{4}$.

Thus, for the purposes of our argument, special edges behave just like vertical ones. We assert next that $X$ contains at most one skew edge. Let $j_{1} j_{1}^{\prime}$ and $j_{2} j_{2}^{\prime}$ be skew edges with $j_{1}<j_{2}$ and $j_{1}+j_{2}$ maximum among the skew edges in $X$.

Observe that $X$ contains no vertical edge $i i^{\prime}$ with $i>j_{2}$ and $i^{\prime}<j_{2}^{\prime}$. Indeed, if there is only one such edge, then it forms an $X-C_{4}$ in $G_{X}$ together with $j_{2} j_{2}^{\prime}$, while if there are at least two such edges, then an $X-C_{4}$ is obtained from a consecutive pair among them.

By a similar argument, $X$ contains neither any vertical edge $i i^{\prime}$ with $j_{1}<i<j_{2}$, nor any vertical edge $i i^{\prime}$ with $j_{2}^{\prime}<i^{\prime}<j_{1}^{\prime}$. It follows that $j_{1} j_{1}^{\prime}$ and $j_{2} j_{2}^{\prime}$ are contained in an $X-C_{4}$ in $G_{X}$, a contradiction which proves that there is at most one skew edge in $X$.

Consequently, $X$ contains a set $Y$ of at least four edges that are vertical or special, as $|X|=5$. Further, $|Y| \neq 5$, so there are exactly four $Y$-copies of $C_{4}$ in $G_{Y}$. Only at most two of these will be affected by the addition of the fifth edge of $X$. Thus, an $X-C_{4}$ persists in $G_{X}$, a contradiction. The proof is complete. 
While the graphs constructed in the proof of Proposition 10 are $C_{4}$-free, they are not cyclically 5-edge-connected. A slight modification of the construction ensures this stronger property, but makes the discussion somewhat more complicated. For this reason, we only described the simpler version.

\section{References}

[1] A. Brandstädt, V. B. Le and J. P. Spinrad, Graph Classes: A Survey, SIAM, Philadelphia, PA, 1999.

[2] G. Brinkmann, J. Goedgebeur, J. Hägglund and K. Markström, Generation and properties of snarks, submitted for publication. Preprint available at arXiv:1206.6690 [math.CO].

[3] M. N. Ellingham, Petersen subdivisions in some regular graphs, Congr. Numer. 44 (1984), 33-40.

[4] J. L. Goldwasser and C.-Q. Zhang, Permutation graphs and Petersen graph, Ars Combin. 51 (1999), 240-248.

[5] H.-J. Lai and C.-Q. Zhang, Hamilton weights and Petersen minors, J. Graph Theory 38 (2001), 197-219.

[6] R. Thomas, Recent excluded minor theorems for graphs, in: Surveys in Combinatorics, Proc. 17th British Combinatorial Conference (Canterbury), London Math. Soc. Lecture Note Ser. 267, Cambridge Univ. Press, Cambridge, 1999, pp. 201-222.

[7] W. T. Tutte, On the algebraic theory of graph colorings, J. Combin. Theory 1 (1966), $15-50$.

[8] C.-Q. Zhang, Integer Flows and Cycle Covers of Graphs, M. Dekker, 1997. 